\title{
Place of the paravertebral block in post-operative analgesia in thoracic surgery
}

\author{
Mohamed Matouk ${ }^{1, *}$, Tahar Ait mouheb ${ }^{2}$, and Nacera Benmouhoub ${ }^{3}$ \\ EPH Kouba Hospital ${ }^{1}$, Algiers, Algeria \\ Liamine Debaghine ${ }^{2}$ Hospital, Algiers, Algeria
}

\begin{abstract}
The thoracic epidural analgesia (TEA) remains the standard gold for analgesic support in thoracic surgery, there is an interesting alternative to epidural analgesia, which is the paravertebral block (PVB). The aim in our study was to assess the value of performing a PVB in the management of postoperative pain in thoracic surgery compared to TEA. Methods: 80 patients were randomized to receive either epidural analgesia $(n=38,10 \mathrm{cc}$ bupivacaine $0.5 \%+10$ Y Sufentanyl then $10 \mathrm{cc}$ Bupivacain $0.1 \%+10$ Y Sufentanyl via a PCA device) or PVB analgesia loss of resistance technique $(n=40,10 \mathrm{cc}$ bupivacaine $0.5 \%+10$ Y Sufentanyl via a PCA device). All patients received standard general anesthesia. The peri-operative parameters studied include standard measurement, EVA scale at rest and mobilization, use of morphinics. Results: there is a significant difference between the two groups and the incidents of puncture were significantly more important for the APDT group. The postoperative pain assessment by EVA did not show a significant difference between TEA and PVB Conclusion: the comparison of PVB to TPDA did not find significant difference in the efficacy of analgesia and the side effects. The BPV could be proposed as a first intention for postoperative analgesia in thoracic surgery.
\end{abstract}

\section{Introduction:}

Thoracic surgery is one of the surgeries leading to severe postoperative pain. Thoracotomy is a surgical approach that generates acute pain of major intensity and for a prolonged period [1-2], but also at a distance with a risk of chronicization [3]. This acute pain, increases the metabolic demand of the operated on and has a direct consequence on its ventilatory mechanics and can induce serious complications. In addition, chronic pain, most often neuropathic [4], is frequent after this type of surgery and is directly related to the intensity of acute postoperative pain [5]. The postoperative analgesia must be optimal in order to ensure early rehabilitation and prevent the onset of chronic pain. A multimodal perioperative analgesia strategy is necessary. Thoracic epidural analgesia (TEA) remains the "gold standard" for analgesic management in this context [6-7].

\section{Materials and methods:}

This are a prospective, descriptive, randomized singleblind study and monocentric carried out within the Thoracic Surgery department of Bab-El-Oued CHU in Algiers over a period of 24 months from October 2012 to October 2014

\subsection{Population:}

Our population consisted of 80 adult patients over the age of 18 , admitted to the service during the study period. These patients were classified ASA I to III and underwent posterolateral thoracotomy with informed consent of patients and agreement of the local ethics committee. Indications are diverse (lung cyst, pneumothorax, tumors, bronchial dilation, bronchogenic cysts, emphysema bubbles).

\subsection{Inclusion criteria:}

Postoperatively, the benefit of epidural analgesia is demonstrated by optimal analgesic coverage, improvement of diaphragmatic contractility, optimization of compliance with respiratory exercises, reduction of respiratory failures, reduction of the length of stay and a decrease in the incidence of chronic pain [3]. However, this technique requires good mastery and exposes in particular to the risk of direct spinal cord injury. The most attractive alternative to an epidural is paravertebral block (BPV) [13]. Recent data in the literature suggest comparable analgesic efficacy between thoracic epidural. The aim of our study was assessing the value of performing a paravertebral block as part of the management of postoperative pain in thoracic surgery in comparison to thoracic epidural analgesia

ASA class I, II, III patients, age $\geq 18$ years old and the patient operated on for an anterolateral thoracotomy.

\subsection{Exclusion criteria:}

Refusal of the analgesia technique by the patient, local infection, allergy to local anesthetics (LA), pleurotomy planned, pleural pocket, patient with a hemostasis disorder or requiring the administration of an anticoagulant preoperatively or, receiving antiplatelet therapy, ASA IV patient and psychiatric pathology or patient with a limitation of his intellectual capacities

\subsection{Randomization:}

was done by random permutation tables, after admission: thoracic epidural group (TEA)and paravertebral block group (PVB). All patients received general anesthesia. Induction was made with propofol 


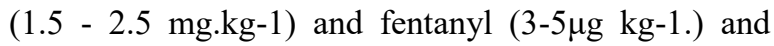
curarization by vecuronium bromide $(0.1 \mathrm{mg} \mathrm{kg}-1)$. No opioid dose supplemental intravenous therapy has not been given after induction. Maintenance was ensured by propofol ( 6 to $12 \mathrm{mg} / \mathrm{kg} / \mathrm{h}$ in the syringe pump) or sevoflurane $(1-2 \%)$ in combination with oxygen. All procedures were performed by surgeons using the same techniques with pose of 1 or 2 chest drains. All patients received postoperative supplemental analgesia with acetaminophen (Paracetamol $\AA$ ) and diclofenac. Analgesia was initiated during surgical closure and maintained for 48 to 72 hours. Thoracic epidural analgesia (TEA): After disinfection, the establishment of the TEA is metameric. Puncture in T6 / T7 or T7 / $\mathrm{T} 8$, using the liquid mandrel loss of strength technique. The TEA catheter is tunneled. The test dose is $03 \mathrm{cc}$ of $2 \%$ adrenaline lidocaine followed by a bolus of $10 \mathrm{cc}$ of $0.5 \%$ bupivacaine combined with $10 \mu \mathrm{g}$ of sufentanyl. Maintenance is carried out by administering to the SAP (Rate: $10 \mathrm{cc} /$ hour) $10 \mathrm{cc}$ of $0.1 \%$ bupivacaine associated with $10 \mu \mathrm{g}$ of sufentanyl upon surgical closure and continued for 48 to $72 \mathrm{~h}$. The puncture of the interspinous space is median with an angle of inclination of the needle of approximately 45 $\circ$. The level of the puncture is: T6 / T7 or T7 / T8. After disinfection and infiltration of the puncture site, the establishment of the PVB is metameric. The puncture is performed at a point 2 to $3 \mathrm{~cm}$ laterally at the level of the spinous process. PVS is addressed by the technique of loss of resistance to the liquid mandrel. The dermatomes concerned from $\mathrm{T} 2$ to $\mathrm{T} 10$, involving a level of puncture in T6 / T7 or T7 / T8, using the liquid mandrel loss of resistance technique. The PVB catheter is tunneled. The test administered dose is $3 \mathrm{cc}$ of $2 \%$ adrenaline lidocaine, followed by a same bolus and maintenance dose as in TEA. Parameters studied include measurement of VAS scale at rest and mobilization, mean arterial pressure, heart rate, SPO2 and quantification of NVPO, technical failures, use of morphinics, and patient and surgeon's satisfaction. Failure of the analgesia technique: the inability to perform the technique, the fall of the secondary catheter or a VAS $\geq 4 \mathrm{~cm}$ not reduced by the increase in the speed or the concentration of LA (use of exclusive morphine). In case of failure of the epidural technique, analgesia by paravertebral block is proposed. If the PVB is ineffective, a wall block is used alone or in combination with spinal morphine pain relief. The statistical study was carried out using Epi Info software version $6 \mathrm{Fr}$

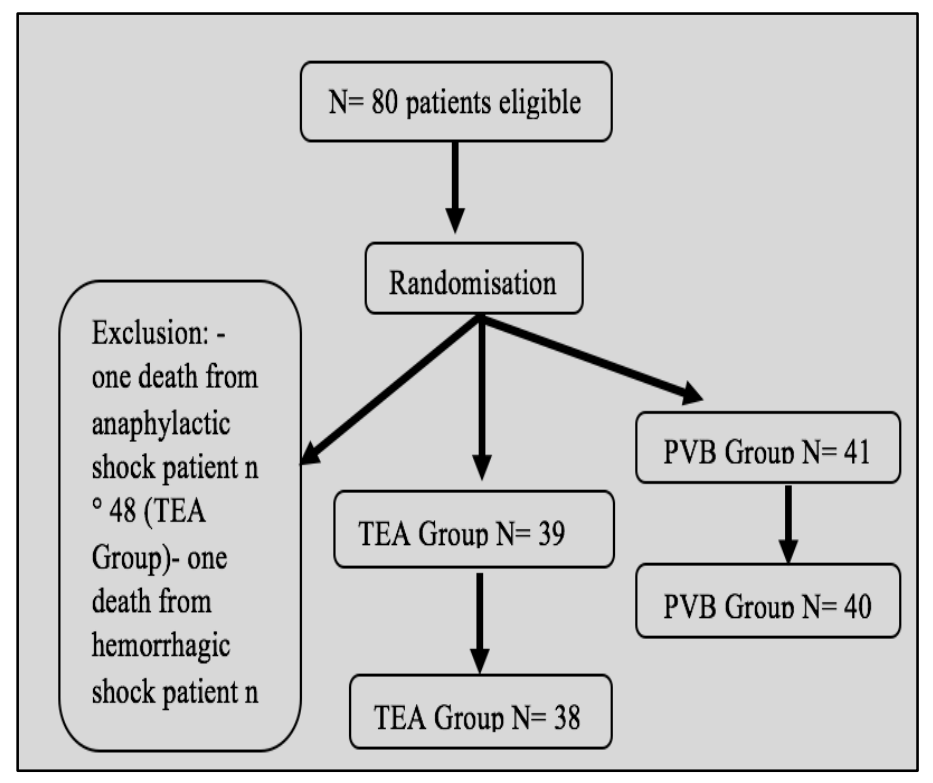

Fig. 1. Flow diagram 
Table 1: Comparison of patient demographics in the TEA and PVB groups.

\begin{tabular}{|c|c|c|c|}
\hline Time & TEA Group $(\mathbf{n}=\mathbf{3 8})$ & PVB Group (n= 40) & $p$ value \\
\hline $00 \mathrm{~h}$ & $3.18 \pm 3.13$ & $3.22 \pm 2.72$ & 0.95 (NSD) \\
\hline $06 \mathrm{~h}$ & $2.55 \pm 2.46$ & $3.59 \pm 2.87$ & 0.40 (NSD) \\
\hline $12 \mathrm{~h}$ & $2.56 \pm 2.51$ & $3.59 \pm 2.87$ & 0.23 (NSD) \\
\hline $18 \mathrm{~h}$ & $2.57 \pm 2.54$ & $3.27 \pm 2.84$ & 0.96 (NSD) \\
\hline $24 \mathrm{~h}$ & $3.03 \pm 2.89$ & $3.00 \pm 2.46$ & 0.91 (NSD) \\
\hline $36 \mathrm{~h}$ & $2.77 \pm 2.74$ & $2.71 \pm 2.23$ & 0.86 (NSD) \\
\hline $48 \mathrm{~h}$ & $2.40 \pm 2.23$ & $2.32 \pm 2.23$ & \\
\hline
\end{tabular}

Table 2: Comparison of patient demographics in the TEA and PVB groups.

\begin{tabular}{|c|c|c|c|}
\hline Variables & $\begin{array}{c}\text { Group A: TEA (n= 38) } \\
\text { Average } \pm \text { SD }\end{array}$ & $\begin{array}{c}\text { Group B: PVB (n= 40) } \\
\text { Average } \pm \text { SD }\end{array}$ & p value \\
\hline Age (years) & $39.03 \pm 15.73$ & $38.66 \pm 16.33$ & 0.91 (NSD) \\
\hline Gender (F/M) & $18 / 21$ & $12 / 29$ & $0.11(\mathrm{NSD})$ \\
\hline Weight (kg) & $60.38 \pm 15.01$ & $67.79 \pm 14.39$ & $0.04(\mathrm{SD})$ \\
\hline Size (m) & $01.66 \pm 0.089$ & $1.70 \pm 0.072$ & $0.34(\mathrm{NSD})$ \\
\hline BMI (kg/m $\left.{ }^{2}\right)$ & $22.46 \pm 3.93$ & $23.47 \pm 4.74$ & $0.01(\mathrm{SD})$ \\
\hline FEV1 (L/S) & $2.42 \pm 0.72$ & $2.88 \pm 0.73$ & $0.94 / 0.51 / 0.52(\mathrm{NSD})$ \\
\hline ASA I/II/III & $25 / 11 / 3$ & $26 / 9 / 6$ & \\
\hline
\end{tabular}

(SD: significant difference - NSD: non-significant difference) - (F : female /M : male) - (L: liter / S : second) With regard to age, sex, body mass index (BMI), ASA score, within group "B" (PVB) weight, height and FEV1 are significantly higher.

The average duration of the intervention, diagnoses and procedures surgical procedures used the two groups remain comparable. For the two techniques, there is no significant difference in concerns the number of attempts and the length of time the catheter is preserved in place. When it comes to the skin-to-space distance, which is more superficial in the PVB technique, there is a significant difference. The puncture incidents were significantly greater for the TEA group. Assessment of pain at rest and during movement: 
Table 3: Evolution over time of the VAS score for pain at rest

\begin{tabular}{|c|c|c|c|}
\hline Time & TEA Group $(\mathbf{n}=\mathbf{3 8})$ & PVB Group (n= 40) & $p$ value \\
\hline $00 \mathrm{~h}$ & $3.18 \pm 3.13$ & $3.22 \pm 2.72$ & 0.95 (NSD) \\
\hline $06 \mathrm{~h}$ & $2.55 \pm 2.46$ & $3.59 \pm 2.87$ & 0.40 (NSD) \\
\hline $12 \mathrm{~h}$ & $2.56 \pm 2.51$ & $3.59 \pm 2.87$ & 0.23 (NSD) \\
\hline $18 \mathrm{~h}$ & $2.57 \pm 2.54$ & $3.27 \pm 2.84$ & 0.96 (NSD) \\
\hline $24 \mathrm{~h}$ & $3.03 \pm 2.89$ & $3.00 \pm 2.46$ & 0.91 (NSD) \\
\hline $36 \mathrm{~h}$ & $2.77 \pm 2.74$ & $2.71 \pm 2.23$ & 0.86 (NSD) \\
\hline $48 \mathrm{~h}$ & $2.40 \pm 2.23$ & $2.32 \pm 2.23$ & \\
\hline
\end{tabular}

Table 4: Evolution over time of the VAS pain score in movement

\begin{tabular}{|c|c|c|c|}
\hline Time & TEA Group (n= 38) & PVB Group (n= 40) & $p$ value \\
\hline $00 \mathrm{~h}$ & $2.87 \pm 1.91$ & $2.88 \pm 2.03$ & 0.98 (NSD) \\
\hline $06 \mathrm{~h}$ & $2.38 \pm 1.37$ & $2.66 \pm 2.12$ & 0.48 (NSD) \\
\hline $12 \mathrm{~h}$ & $2.46 \pm 1.41$ & $2.17 \pm 2.21$ & 0.97 (NSD) \\
\hline $18 \mathrm{~h}$ & $2.38 \pm 1.62$ & $2.37 \pm 1.88$ & 0.13 (NSD) \\
\hline $24 \mathrm{~h}$ & $2.41 \pm 1.77$ & $1.83 \pm 1.66$ & 0.51 (NSD) \\
\hline $36 \mathrm{~h}$ & $2.03 \pm 1.55$ & $1.80 \pm 1.57$ & 0.51 (NSD) \\
\hline $48 \mathrm{~h}$ & $1.92 \pm 1.53$ & $1.50 \pm 1.54$ & \\
\hline
\end{tabular}

Evaluation of postoperative pain by VAS did not show any significant difference between the two groups. Sensory levels in both groups were similar from T2-T3 to higher level than T8-T9 at lower level.

\subsection{Adverse events:}

MAP, SPO2, Mean HR, values were comparable in the two groups during the first 48 hours postoperatively.

\subsection{Technical failures, side effects (PONV) and use of morphine}

No significant difference was observed between the two groups of patients with regard to technical failures, side effects (PONV) and use of opioids.

\subsection{Patient and surgeon satisfaction:}

Patient and surgeon satisfaction were better in the APDT group, but the difference was not significant

\section{Discussion:}

The origin of post-thoracotomy pain is complex [120-21). In a multimodal setting, several analgesia techniques have been proposed to improve the peri and postoperative care of operated patients by thoracotomy [22-23]. Our study aimed to assess the value of performing a paravertebral block as part of the management of postoperative pain in thoracic surgery compared to epidural thoracic analgesia. 
The paravertebral block was performed as a first intention alongside the TEA [6-13-24-25]. The population of the two groups TEA and PVB was comparable, according to the demographic criteria, duration of intervention, pathology and surgical protocol. The difference in weight, height and FEV1, which is greater for the PVB group, is probably linked to a selection bias. Technical characteristics PVB was significantly more superficial than TEA in our study, as in Michelle D. Greene et al [24]. As found by De Cosmo et al [26], minor puncture incidents were significantly more common in the APDT group with vagal discomfort and reflux of blood into the catheter, but without serious consequences for the patients. These incidents are probably related to the difficulty thoracic epidural puncture technique. The shelf life of the catheter was 4 to 5 days in the two groups, corresponding to the retention time of the thoracic drains in our department, contrary to the data which recommends 48 to $72 \mathrm{~h}$ [27-28]

During the performance of the BPV, the correct location of the catheter was checked by a chest $\mathrm{x}$ ray after opacification in more than $80 \%$ of cases, depending on the availability of the contrast medium, even if the lack of correlation between opacification and efficacy is emphasized in the literature. [29-30-31]. Pain scores were low (VAS $<4$ ) in both groups, both at mobilization and at rest, for 4 to 5 days, with no significant difference, despite better motion analgesia with TEA, requiring additional boluses of LA in the PVB group. Sensory levels in both groups were similar from T2-T3 higher level to T8-T9on the lower level, in agreement with those found in several studies. [1332]. Our results are in agreement with the data in the literature, in particular the study of Perttunen K. [34] unlike the studies by Richardson [35] and Pintaric [36], due to the low doses and concentrations of LA in our work. Respiratory effects: We did not note any desaturation below $96 \%$ in the two groups. Note, however, that Richard-son et. al. in a study in 1999 [35] observed that the pulmonary function was significantly better preserved in the PVB group where the values of $\mathrm{SPO} 2$ were the highest and the respiratory complications practically non-existent. This result would be linked to a greater use of morphine in the TEA group which had a negative effect on the spirometric performance of the patients. The use of opioids was greater in the PVB group, but without significant difference. This result is in agreement with the conclusions of the meta-analysis by Ding X. et al. in 2014 [32] and work by Dhole S. et al. (2001) [37]. Unlike the work of Richardson J. et al. (1999) [35] where the use of larger amounts of morphine in the TEA group resulted in an increased incidence of negative effects on respiratory function. Assessment of patient and surgeon satisfaction is rare in Literature. In our study, patient and surgeon satisfaction scores were good, as in the study of From Cosmo G et al. (2002) [26]. In our study, the comparison of PVB to TEA did not find any significant difference in both the efficacy of analgesia and the side effects. Study objectives were met The PVB seems efficient in daily practice since the installation time was fast with efficiency of the block during clinical tests, easy to perform, successful with a lower number of attempts. PVB could be offered as a first-line treatment for postoperative analgesia in thoracic surgery.

Limitations of the study: Like all studies, our work has limitations: Its realization without double blind, with probable bias of selections. Lack of ultrasound guidance. The reason for not using ultrasound was: Compliance with the protocol, recommending the execution in the procedure of the loss of resistance technique for both PVB and TEA. The unavailability of an ultrasound machine at the time of the study. The heterogeneity and noncoding of para-vertebral ultrasound guidance at the time of the study [39-40].

\section{$\underline{\text { References }}$}

1. RG.Soto, ES.Fu Acute pain management for patients undergoing thoracotomy. 75(4) : p 1349-57. Ann.Thorac. Surg Apr; (2003)

2. H.Kehlet, TS.Jensen, CJ Woolf. Persistent postsurgical pain: risk factorsand prevention. 13; 367 (9522): p 1618-25. Lancet. May (2006)

3. SS.Reuben, L. Yalavarthy. Preventing the development of chronic pain afte rthoracic surgery.; 22 (6): p 890-903. J. Cardiothorac. Vasc. Anesth. Dec (2008)

4. RD. Searle, MI. Bennett. Neuropathic pain after thoracic surgery. Pain. 152 (4): p 958. 2011Apr;

5. J. Katz, M Jackson, BP. Kavanagh, AN. Sandler. Acute pain after thoracicsurgery predicts long-term post-thoracotomy pain; 12(1): p 50 5. . Clin J Pain (1996)

6. GP. Joshi, F.Bonnet, R. Shah, RC.Wilkinson, Camu F, Fischer B, Neuge. A systematic review of randomized trials evaluating regional techniques for postthoracotomy analgesia. Anesth. Analg.; 107 (3): p 1026-40. (2008)

7. M. Sentürk, PE . Ozcan, GK. Talu, Kiyan E, Camci E, Ozyalçin S, Dilege S, Pembeci K. The effects of three different analgesia techniques on long-term posthoracotomy pain. Anesth Analg; 94: p 11-5. 2002 
8. C1. Bauer, Hentz JG, Ducrocq X, Meyer N, M Oswald-Mammosser, A Steib, JP Dupeyron. Lung function after lobectomy: A randomized, double-blind trial comparing thoracic epidural ropivacaine/sufentanil and intraveinous morphine for patient- controlled analgesia. Anesth Analg; 105: p 238-44. (2007)

9. DM. Pöpping, N.Elia, E.Marret, C.Remy, MR.Tramèr . Protective effects of epidural analgesia on pulmonary complications after abdominal and thoracic surgery: a metaanalysis. Arch Surg; 143: p 990-9. (2008)

10. A1. Rodgers, N.Walker, S.Schug, A.McKee, H.QKehlet, van Zundert A, Sage D, Futter M, Saville G, Clark T, MacMahon S. Reduction of postoperative mortality and morbidity with epidural or spinal anaesthesia: results from overview of randomised trials. BMJ. 16;321(7275): p 1493. (2000)

11. WS. Beattie, NH. Badner, P. Choi. Epidural analgesia reduces postoperative myocardial infarction: A meta-analysis. Anesth Analg; 93: p 853-8. (2001)

12. T. Oka, Y.Ozawa, Y.Ohkubo .Thoracic epidural bupivacaine attenuates supraventricular tachyarrythmias after pulmonary resection. Anesth Analg; 93: p 2539. (2001)

13. RG. Davies, PS. Myles, JM. Graham. $A$ comparison of the analgesic efficacy and sideeffects of paravertebral vs epidural blockade for thoracotomy-a systematic review and metaanalysis of randomized trials. Br J Anaesth; 96: p 418-426. (2006)

14. J. Richardson, Fin-de-siecle renaissance of paravertebral analgesia. Pain Rev; 4:p 15971(1997)

15. RS. Atkinson, Rushman GB, Lee JA. $A$ Synopsis of Anaesthesia. Bristol, IOP Publishing Limited, p 628-9 (1987)

16. Lönnqvist, P. A., and Jonathan Richardson. "Use of paravertebral blockade in children." Techniques in Regional Anesthesia and Pain Management 3.3 (1999): 184-188.

17. Eason MJ and Wyatt R. Paravertebral thoracic block - a reappraisal. Anaesthesia ; Volume 34, Issue 7: p 638-642.(1979)

18. Tiippana E, Nilsson E, Kalso E. Postthoracotomy pain after thoracic epidural analgesia: a prospective follow-up study. Acta Anaesthesiol Scand. Apr; 47(4) : p433-8. (2003)

19. Mesbah A, Yeung J, Gao F Pain after thoracotomy. BJA Education, Vol 16, Issue 1, 1 January (2016)

20. Diana Nordquist and Thomas M. Halaszynski Perioperative multimodal anesthesia using regional techniques in the aging surgical patient. Pain Research and Treatment Volume, Article ID 902174, 13 p. (2014)

21. Bottiger BA, Esper SA, Stafford-Smith M. Pain management strategies for thoracotomy and thoracic pain syndromes. Semin Cardiothorac Vasc Anesth. Mar; 18(1): p 4556. (2014)

22. SQM Tighe, Michelle D Greene and Nirmal Rajadurai Paravertebral block.in Continuing Education in Anaesthesia, Critical Care \& Pain Published on behalf of The British J. of Anaesth. 105, p: 133-137. (2010)

23. Dango S, Harris S, Offner K, Hennings E, Priebe HJ, Buerkle H, Passlick B, Loop T. Combined paravertebral and intrathecal vs thoracic epidural analgesia for postthoracotomy pain relief. Br J Anaesth. Mar; 110(3): p443-9. (2013)

24. G De Cosmo P Aceto; E Campanale; E Congedo; A Clemente; A Mascia; $P$ GranoneComparison between epidural and paravertebral intercostal nerve block with ropivacaine after thoracotomy: effects on pain relief, pulmonary function and patient satisfaction. Acta Medica Romana; 40, Part 4: p 340-347. (2002)

25. Kotze, A., A. Scally, and S. Howell. "Efficacy and safety of different techniques of paravertebral block for analgesia after thoracotomy: a systematic review and metaregression."

26. Raveglia F, Rizzi A, Leporati A, Di Mauro P, Cioffi U, Baisi A. Analgesia in patients undergoing. A undergoing thoracotomy: epidural versus paravertebral technique. randomized, double-blind, prospective study.

J Thorac Cardiovasc Surg.; 147(1): p469-73 (2014)

27. Marhofer D, Marhofer P, Kettner SC, Fleischmann E, Prayer D, Schernthaner M, Lackner E, Willschke H, Schwetz P, Zeitlinger M. Magnetic resonance imaging analysis of the spread of local anesthetic solution after ultrasound-guided lateral thoracic paravertebral blockade: a volunteer study. Anesthesiology. May;118(5):p 1106-12. (2013)

28. Purcell-Jones, G., Pither, C. E., Justins, D. M. Paravertebral somatic nerve block: a clinical, radiographic, and computed tomographic study in chronic pain patients. Anesthesia \& Analgesia; 68 (1): p 32-39. (1989)

29. C. Luyet, A. Siegenthaler, Z. Szucs-Farkas, G, Hummel, U. Eichelberger, A. Vogt The location of paravertebral catheters placed using 
the landmark technique.Anaesthesia ; 67 (12): p 1321-1326. (2012)

30. Ding X, Jin S, Niu X, Ren H, Fu S, Li Q. $A$ comparison of the analgesia efficacy and side effects of paravertebral compared with epidural blockade for thoracotomy: an updated meta-analysis. PLoS One; 9(5): p e96233. (2014)

31. Yeung JH, Gates S, Naidu BV, Wilson MJ, Gao Smith F. Paravertebral block versus thoracic epidural for patients undergoing thoracotomy. Cochrane Database Syst Rev. Feb 21; 2:CD009121. (2016)

32. Perttunen K, Nilsson E, Heinonen J, Hirvisalo EL, Salo JA, Kalso E.Extradural, paravertebral and intercostal nerve blocks for post-thoracotomy pain. Br J Anaesth ; 75(5): p541-7. (1995)

33. Richardson J, Sabanathan S, Jones J, Shah RD, Cheema S, Mearns AJ. A prospective, randomized comparison of preoperative and continuous balanced epidural or paravertebral bupivacaine on post-thoracotomy pain,pulmonary function and stress responses. Br J Anaesth.; 83(3): p387-92. (1999)

34. Pintaric TS, Potocnik I, Hadzic A, Stupnik T, Pintaric M, Novak Jankovic V. Comparison of continuous thoracic epidural with paravertebral block on perioperative analgesia and hemodynamic stability in patients having open lung surgery. Reg Anesth Pain Med; 36 (3): p 256-60. (2011)

35. Dhole S, Mehta Y, Saxena H, Juneja R, Trehan N. Comparison of continuous thoracic epidural and paravertebral blocks for postoperative analgesia after minimally invasive direct coronary artery bypass surgery. J Cardiothorac Vasc Anesth.; 15(3): p288-92. (2001)

36. AC.Krediet, N Moayeri, van Geffen GJ, Bruhn J, Renes S, PE. Bigeleisen, GJ. Groen. Different approaches to ultrasound-guided thoracic paravertebral block. Anesthesiology; 123 (2): p459-474. (2015)

37. Pace MM, Sharma B, Anderson-Dam J, Fleischmann K, Warren L, Stefanovich P. Ultrasound-guided thoracic paravertebral blockade: a retrospective study of the incidence of complications. Anesth Analg.; 122 (4): p 1186-91. (2016) 